\title{
Review of: "How Effective Is Traffic and Production Restriction for Air Pollution? Evidence From a Quasi- natural Experiment in China"
}

\author{
Rui Bao ${ }^{1}$ \\ 1 Peking University
}

Potential competing interests: The author(s) declared that no potential competing interests exist.

\section{Comments on "How Effective Is Traffic and Production Restriction for Air Pollution? Evidence From a Quasi-natural Experiment in China"}

The above-mentioned manuscript examines the association between traffic and production restriction and air pollution. The topic of this study is hot. However, the study is not sufficient novel, in terms of methodology and scientific finding. Similar results have been extensively reported in a series of newly published papers in various journals. The method used in this study was not new and the major findings are well known by now. Anyway, this paper is well written. Nevertheless, I have certain concerns, with the bigger ones concerning the causal inference:

1. To the best of my understanding Wuhan was locked down on 23 January 2020. A few days later, similar quarantine measures were imposed on its neighboring cities. Numerous studies have proved that spillover effects and regional effects exist in air pollution. Is there spatial spillover effect in air pollution between Wuhan and its neighboring cities? If any, can this affect your results and in which direction? Is it possible to consider a spatial analysis to capture the inherent spatial dependence?

2. Another factor that could affect the observed relationship is the time trends and lagged effects that may affect daily air quality. On the one hand, China has moved aggressively to reduced air pollution in recent years. Air pollution is decreasing year by year. Your results in Table 2 may be invalidated without considering the yearly trends in air pollution. On the other hand, temporal dependence on daily air pollution (i.e. time series autocorrelation) may also invalidates your results in Table 4. For example, today's air pollution level may be affected by its historical pollution level of yesterday or earlier days. And this temporal dependence is varied by specific air pollutants. Could you have a lagged term in time to captures this? Another fact needs to be considered is that, with the coming of spring, temperature rising, the demand of heating will decrease. Theoretically, Wuhan is also expected to experience a reduction in air pollution.

3. Although the author mentioned that they implemented several sensitivity analyses, I have low confidence in the reported estimates results. Please offer more robustness check. 
4. Basically, the authors should report the t or SE (standard error) statistics of the estimated coefficients and the number of observations in each regression. The validation of RD estimation is highly dependent on the sample size.

5. The literature review regarding is not sufficient. The following published articles should also be included and discussed:

- He, G., Pan, Y., Tanaka, T., 2020. The short-term impacts of COVID-19 lockdown on urban air pollution in China. Nature Sustainability.

- Fu, S., Gu, Y., 2017. Highway toll and air pollution: Evidence from Chinese cities. Journal of Environmental Economics \& Management.

- Bao, R., Zhang, A., 2020. Does lockdown reduce air pollution? Evidence from 44 cities in northern China. Sci. Total Environ. 731, 139052. 